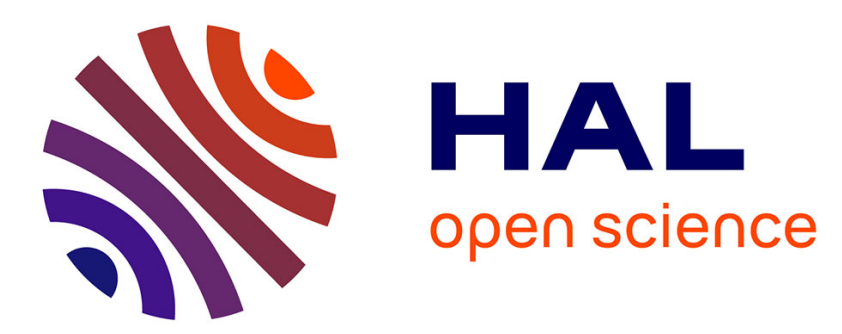

\title{
Abstract sounds and their applications in audio and perception research
}

Adrien Merer, Sølvi Ystad, Richard Kronland-Martinet, Mitsuko Aramaki

\section{To cite this version:}

Adrien Merer, Sølvi Ystad, Richard Kronland-Martinet, Mitsuko Aramaki. Abstract sounds and their applications in audio and perception research. Sølvi Ystad, Mitsuko Aramaki, Richard KronlandMartinet, Kristoffer Jensen. Exploring music contents, Springer Verlag Berlin Heidelberg, pp.176-187, 2011, Lecture Notes in Computer Science, 978-3-642-23125-4. hal-00727560

\section{HAL Id: hal-00727560 https://hal.science/hal-00727560}

Submitted on 3 Sep 2012

HAL is a multi-disciplinary open access archive for the deposit and dissemination of scientific research documents, whether they are published or not. The documents may come from teaching and research institutions in France or abroad, or from public or private research centers.
L'archive ouverte pluridisciplinaire HAL, est destinée au dépôt et à la diffusion de documents scientifiques de niveau recherche, publiés ou non, émanant des établissements d'enseignement et de recherche français ou étrangers, des laboratoires publics ou privés. 


\title{
Abstract sounds and their applications in audio and perception research
}

\author{
Adrien Merer ${ }^{1}$, Sølvi Ystad $^{1}$, Richard Kronland-Martinet ${ }^{1}$, and Mitsuko \\ Aramaki ${ }^{2,3}$ \\ ${ }^{1} \mathrm{CNRS}$ - Laboratoire de Mécanique et d'Acoustique, \\ $31 \mathrm{ch}$. Joseph Aiguier, Marseille, France \\ ${ }^{2}$ CNRS - Institut de Neurosciences Cognitives de la Méditerranée, \\ $31 \mathrm{ch}$. Joseph Aiguier, Marseille, France \\ ${ }^{3}$ Université Aix-Marseille, 38 bd. Charles Livon, Marseille, France \\ \{merer, ystad, kronland\}@lma.cnrs-mrs.fr \\ aramaki@incm.cnrs-mrs.fr
}

\begin{abstract}
Recognition of sound sources and events is an important process in sound perception and has been studied in many research domains. Conversely sounds that cannot be recognized are not often studied except by electroacoustic music composers. Besides, considerations on recognition of sources might help to address the problem of stimulus selection and categorization of sounds in the context of perception research. This paper introduces what we call abstract sounds with the existing musical background and shows their relevance for different applications.
\end{abstract}

Key words: abstract sound, stimuli selection, acousmatic

\section{Introduction}

How do sounds convey meaning? How can acoustic characteristics that convey the relevant information in sounds be identified? These questions interest researchers within various research fields such as cognitive neuroscience, musicology, sound synthesis, sonification, etc. Recognition of sound sources, identification, discrimination and sonification deal with the problem of linking signal properties and perceived information. In several domains (linguistic, music analysis), this problem is known as "semiotics" [21]. The analysis by synthesis approach [28] has permitted to understand some important features that characterize the sound of vibrating objects or interaction between objects. A similar approach was also adopted in [13] where the authors use vocal imitations in order to study human sound source identification with the assumption that vocal imitations are simplifications of original sounds that still contain relevant information.

Recently, there has been an important development in the use of sounds to convey information to a user (of a computer, a car, etc.) within a new research community called auditory display [19] which deals with topics related to sound design, sonification and augmented reality. In such cases, it is important to use 
sounds that are meaningful independently of cultural references taking into account that sounds are presented through speakers concurrently with other audio/visual information.

Depending on the research topics, authors focused on different sound categories (i.e. speech, environmental sounds, music or calibrated synthesized stimuli). In [18], the author proposed a classification of everyday sounds according to physical interactions from which the sound originates. When working within synthesis and/or sonification domains, the aim is often to reproduce the acoustic properties responsible for the attribution of meaning and thus, sound categories can be considered from the point of view of semiotics i.e. focusing on information that can be gathered in sounds.

In this way, we considered a specific category of sounds that we call "abstract sounds". This category includes any sound that cannot be associated with an identifiable source. It includes environmental sounds that cannot be easily identified by listeners or that give rise to many different interpretations depending on listeners and contexts. It also includes synthesized sounds, and laboratory generated sounds if they are not associated with a clear origin. For instance, alarm or warning sounds cannot be considered as abstract sounds. In practice, recordings with a microphone close to the sound source and some synthesis methods like granular synthesis are especially efficient for creating abstract sounds. Note that in this paper, we mainly consider acoustically complex stimuli since they best meet our needs in the different applications (as discussed further).

Various labels that refer to abstract sounds can be found in the literature: "confused" sounds [6], "strange" sounds [36], "sounds without meaning" [16]. Conversely, [34] uses the term "source-bonded" and the expression "source bonding" for the "The natural tendency to relate sounds to supposed sources and causes".

Chion introduced "acousmatic sounds" [9] in the context of cinema and audiovisual applications with the following definition: "sound one hears without seeing their originating cause - an invisible sound source" (for more details see section $2)$.

The most common expression is "abstract sounds" $[27,14,26]$ particularly within the domain of auditory display, when concerning "earcons" [7]. "Abstract" used as an adjective means "based on general ideas and not on any particular real person, thing or situation" and also "existing in thought or as an idea but not having a physical reality" . For sounds, we can consider another definition used for art "not representing people or things in a realistic way" ${ }^{\text {. Abstract as a }}$ noun is "a short piece of writing containing the main ideas in a document" 1 and thus share the ideas of essential attributes which is suitable in the context of semiotics. In [4], authors wrote: "Edworthy and Hellier (2006) suggested that abstract sounds can be interpreted very differently depending on the many possible meanings that can be linked to them, and in large depending on the surrounding environment and the listener."

\footnotetext{
${ }^{1}$ Definitions from http://dictionary.cambridge.org/
} 
In fact, there is a general agreement for the use of the adjective "abstract" applied to sounds that express both ideas of source recognition and different possible interpretations.

This paper will first present the existing framework for the use of abstract sounds by electroacoustic music composers and researchers. We will then discuss some important aspects that should be considered when conducting listening tests with a special emphasis on the specificities of abstract sounds. Finally, three practical examples of experiments with abstract sounds in different research domains will be presented.

\section{The acousmatic approach}

Even if the term "abstract sounds" was not used in the context of electroacoustic music, it seems that this community was one of the first to consider the issue related to the recognition of sound sources and to use such sounds. In 1966, P. Schaeffer, who was both a musician and a researcher, wrote the Traité des objets musicaux [29], in which he reported more than ten years of research on electroacoustic music. With a multidisciplinary approach, he intended to carry out fundamental music research that included both "Concrète" 2 and traditional music. One of the first concepts he introduced was the so called "acousmatic" listening, related to the experience of listening to a sound without paying attention to the source or the event. The word "acousmatic" is at the origin of many discussions, and is now mainly employed in order to describe a musical trend. Discussions about "acousmatic" listening was kept alive due to a fundamental problem in Concrète music. Indeed, for music composers the problem is to create new meaning from sounds that already carry information about their origins. In compositions where sounds are organized according to their intrinsic properties, thanks to the acousmatic approach, information on the origins of sounds is still present and interacts with the composers' goals.

There was an important divergence of points of view between Concrète and Elektronische music (see [10] for a complete review), since the Elektronische music composers used only electronically generated sounds and thus avoided the problem of meaning [15]. Both Concrète and Elektronische music have developed a research tradition on acoustics and perception, but only Schaeffer adopted a scientific point of view. In [11], the author wrote: "Schaeffer's decision to use recorded sounds was based on his realization that such sounds were often rich in harmonic and dynamic behaviors and thus had the largest potential for his project of musical research". This work was of importance for electroacoustic musicians, but is almost unknown by researchers in auditory perception, since there is no published translation of his book except for concomitant works [30]

\footnotetext{
2 The term "concrete" is related to a composition method which is based on concrete material i.e recorded or synthesized sounds, in opposition with "abstract" music which is composed in an abstract manner i.e from ideas written on a score, and become "concrete" afterwards.
} 
and Chion's Guide des objets musicaux ${ }^{3}$. As reported in [12], translating Schaeffer's writing is extremely difficult since he used neologisms and very specific meanings of french words. However, recently has been a growing interest in this book and in particular in the domain of music information retrieval, for the morphological sound description $[27,26,5]$. Authors indicate that in the case of what they call "abstract" sounds, classical approaches based on sound source recognition are not relevant and thus base their algorithms on Schaeffer's morphology and typology classifications.

Morphology and typology have been introduced as analysis and creation tools for composers as an attempt to construct a music notation that includes electroacoustic music and therefore any sound. The typology classification (cf. figure 1 ) is based on a characterization of spectral (mass) and dynamical (facture ${ }^{4}$ ) "profiles" of with respect to their complexity and consists of twenty-eight categories. There are nine central categories of "balanced" sounds for which the variations are neither too rapid and random nor too slow or nonexistent. Those nine categories included three facture profiles (sustained, impulsive or iterative) and three mass profiles (tonic, complex and varying). On both sides of the "balanced objects" in the table, there are nineteen additional categories for which mass and facture profiles are very simple/repetitive or vary a lot.

Note that some automatic classification methods are available [26]. In [37] the authors proposed an extension of Schaeffer's typology that includes graphical notations.

Since the 1950s, electroacoustic music composers have addressed the problem of meaning of sounds and provided an interesting tool for classification of sounds with no a priori differentiation on the type of sound. For sound perception research, a classification of sounds according to these categories may be useful since they are suitable for any sound. The next section will detail the use of such classification for the design of listening tests.

\section{Design of listening tests using abstract sounds}

The design of listening tests is a fundamental part of sound perception studies and implies considerations of different aspects of perception that are closely related to the intended measurements. For instance, it is important to design calibrated stimuli and experimental procedures to control at best the main factors that affect the subjects' evaluations. We propose to discuss such aspects in the context of abstract sounds.

\subsection{Stimuli}

It is common to assume that perception differs as a function of sound categories (e.g. speech, environmental sounds, music). Even more, these categories

\footnotetext{
3 Translation by J.Dack available at http://www.ears.dmu.ac.uk/spip.php?page= articleEars\&id_article $=3597$

${ }^{4}$ As discussed in [12] even if facture is not a common English word, there is no better translation from French
} 


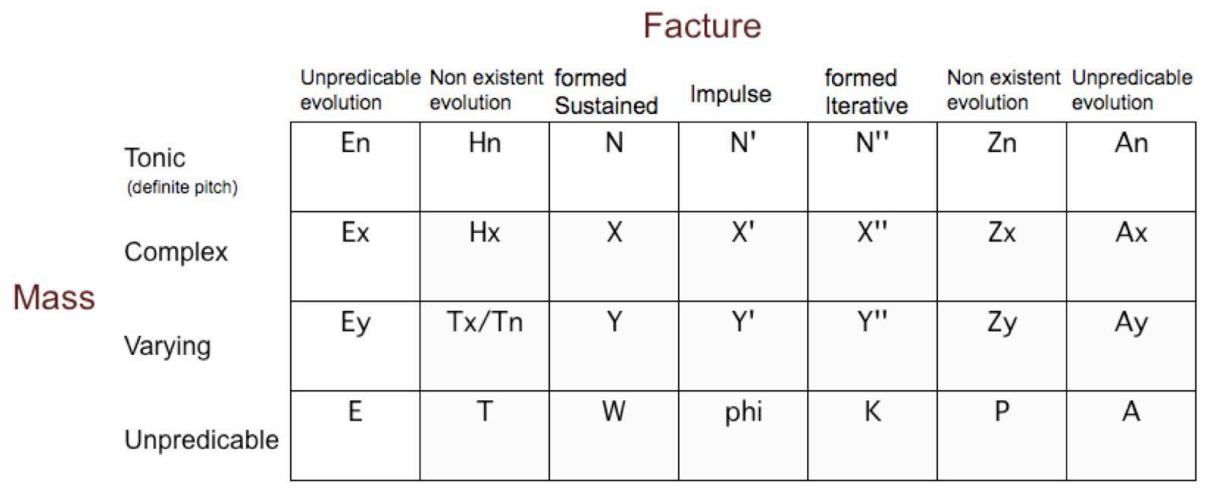

Fig. 1. Schaeffer's typology. Note that some column labels are redundant since the table must be read from center to borders. For instance, the "Non existent evolution" column in the right part of the table corresponds to endless iterations whereas the "Non existent evolution" column in the left part concerns sustained sounds (with no amplitude variations).

Translation from [12]

are underlying elements defining a research area. Consequently, it is difficult to determine a general property of human perception based on collected results obtained from different studies. For instance, results concerning loudness conducted on elementary synthesized stimuli (sinusoids, noise, etc.) cannot be directly adapted to complex environmental sounds as reported by [31]. Furthermore, listeners' judgements might differ for sounds belonging to a same category. For instance, in the environmental sound category, [14] have shown specific categorization strategies for sounds that involve human activity.

When there is no hypothesis regarding the signal properties, it is important to gather sounds that present a large variety of acoustic characteristics as discussed in [33]. Schaeffer's typology offers an objective selection tool than can help the experimenter to construct a very general sound corpus representative of most existing sound characteristics by covering all the typology categories. As a comparison, environmental sounds can be classified only in certain rows of Schaeffer's typology categories (mainly the "balanced" objects). Besides, abstract sounds may constitute a good compromise in terms of acoustic properties between elementary (sinusoids, noise, etc.) and ecological (speech, environmental sounds and music) stimuli.

A corpus of abstract sounds can be obtained in different ways. Many databases available for audiovisual applications contain such sounds (see [33]). Different synthesis techniques (like granular or FM synthesis, etc.) are also efficient to create abstract sounds. In [16] and further works $[38,39]$, the authors presented some techniques to transform any recognizable sound into an abstract sound, 
preserving several signal characteristics. Conversely, many transformations drastically alter the original (environmental or vocal) sounds when important acoustic attributes are modified. For instance, [25] has shown that applying high and low-pass filtering influence the perceived naturalness of speech and music sounds. Since abstract sounds do not convey univocal meaning, it is possible to use them in different ways according to the aim of the experience. For instance, a same sound corpus can be evaluated in different contexts (by drawing the listener's attention to certain evocations) in order to study specific aspects of the information conveyed by the sounds. In particular, we will see how the same set of abstract sounds was used in 2 different studies described in sections 4.3 and 4.1.

\subsection{Procedure}

To control the design of stimuli, it is important to verify in a pre-test that the evaluated sounds are actually "abstract" for most listeners. In a musical context, D. Smalley [35] has introduced the expression "surrogacy" level (or degree) to quantify the ease of source recognition. This level is generally evaluated by using identification tasks. In [6], the authors describe three methods: 1) Free identification tasks that consists of associating words or any description with sounds [2]. 2) Context-based ratings, which are comparisons between sounds and other stimuli. 3) Attribute rating, which is a generalization of the semantic differential method. The third method may be the most relevant since it provides graduated ratings on an unlimited number of scales. In particular, we will see in section 4.3 that we evaluated the degree of recognition of abstract sounds ("the sound is easily recognizable or not") by asking listeners to use a non graduated scale from "not recognizable" to "easily recognizable".

Since abstract sounds are not easily associated with a source (and to the corresponding label), they can also be attributed to several meanings that may depend on the type of experimental procedure and task. In particular, we will see that it is possible to take advantage of this variability of meaning to highlight for example differences between groups of listeners as described in section 4.1.

\subsection{Type of listening}

In general, perception research distinguishes analytic and synthetic listening. Given a listening procedure, subjects may focus on different aspects of sounds since different concentration and attention levels are involved. From a different point of view, [17] introduced the terms "everyday listening" (as opposed to "musical listening") and argued that even in the case of laboratory experiences, listeners are naturally more interested in sound source properties than in intrinsic properties and therefore use "everyday listening". [29] also introduced different types of listening ("hearing", "listening", "comprehending", "understanding") and asserted that when listening to a sound we switch from one type of listening to another. Even if different points of view are used to define the different types of listening, they share the notions of attentional direction and intention when 
perceiving sounds. Abstract sounds might help listeners to focus on intrinsic properties of sound and thus to adopt musical listening.

Another aspect that could influence the type of listening and therefore introduce variability in responses is the coexistence of several streams in a sound ${ }^{5}$. If a sound is composed of several streams, listeners might alternatively focus on different elements which cannot be accurately controlled by the experimenter. Since abstract sounds have no univocal meaning to be preserved, it is possible to proceed to transformations that favour one stream (and alter the original meaning). This is not the case for environmental sound recordings for instance, since transformations can make them unrecognizable. Note that classification of sounds with several streams according to Schaeffer's typology might be difficult since they present concomitant profiles associated with distinct categories.

\section{Potentials of abstract sounds}

As described in section 2, potentials of abstract sounds was initially revealed in the musical context. In particular, their ability to evoke various emotions was fully investigated by electroacoustic composers. In this section, we describe how abstract sounds can be used in different contexts by presenting studies linked to three different research domains, i.e. sound synthesis, cognitive neuroscience and clinical diagnosis. Note that we only aim at giving an overview of some experiments that use abstract sounds, in order to discuss the motivations behind the different experimental approaches. Details of the material and methods can be found in the referred articles in the following sections.

The three experiments partially shared the same stimuli. We collected abstract sounds provided by electroacoustic composers. Composers constitute an original resource of interesting sounds since they have thousands of specially recorded or synthesized sounds, organized and indexed to be included in their compositions. From these databases, we selected a set of 200 sounds $^{6}$ that best spread out in the typology table proposed by Schaeffer (cf. tab 1). A subset of sounds was finally chosen according to the needs of each study presented in the following paragraphs.

\subsection{Bizarre and familiar sounds}

Abstract sounds are not often heard in our everyday life and could even be completely novel for listeners. Therefore, they might be perceived as "strange" or "bizarre". As mentioned above, listeners" judgements of abstract sounds are

\footnotetext{
${ }^{5}$ Auditory streams have been introduced by Bregman [8], and describe our ability to group/separate different elements of a sound

${ }^{6}$ Some examples from [23] are available at http://www.sensons.cnrs-mrs.fr/ CMMR07_semiotique/
} 
highly subjective. In some cases, it is possible to use this subjectivity to investigate some specificities of human perception and in particular, to highlight differences of sound evaluations between groups of listeners. In particular, the concept of "bizarre" is one important element from standard classification of mental disorders (DSM - IV) for schizophrenia [1] pp. 275. An other frequently reported element is the existence of auditory hallucinations ${ }^{7}$, i.e. perception without stimulation. From such considerations, we explored the perception of bizarre and familiar sounds in patients with schizophrenia by using both environmental (for their familiar aspect) and abstract sounds (for their bizarre aspect). The procedure consisted in rating sounds on continuous scales according to a perceptual dimension labelled by an adjective (by contrast, classical differential semantic uses an adjective and an antonym to define the extremes of each scale). Sounds were evaluated on six dimensions along linear scales: "familiar", "reassuring", "pleasant", "bizarre", "frightening", "invasive" 8 . Concerning the abstract sound corpus, we chose 20 sounds from the initial set of 200 sounds by a pre-test on seven subjects and selected sounds that best spread in the space of measured variables (the perceptual dimensions). This preselection was validated by a second pre-test on fourteen subjects that produced similar repartition of the sounds along the perceptual dimensions.

Preliminary results showed that the selected sound corpus made it possible to highlight significant differences between patients with schizophrenia and control groups. Further analysis and testing (for instance brain imaging techniques) will be conducted in order to better understand these differences.

\subsection{Reduction of linguistic mediation and access to different meanings}

Within the domain of cognitive neuroscience, a major issue is to determine whether similar neural networks are involved in the allocation of meaning for language and other non-linguistic sounds. A well-known protocol largely used to investigate semantic processing in language, i.e. the semantic priming paradigm [3], has been applied to other stimuli such as pictures, odors and sounds and several studies highlighted the existence of a conceptual priming in a nonlinguistic context (see [32] for a review). One difficulty that occurs when considering non-linguistic stimuli, is the potential effect of linguistic mediation. For instance watching a picture of a bird or listening to the song of a bird might automatically activate the verbal label "bird". In this case, the conceptual priming cannot be considered as purely non-linguistic because of the implicit naming induced by the stimulus processing. Abstract sounds are suitable candidates to weaken this problem, since they are not easily associated with a recognizable source. In [32], the goals were to determine how a sense is attributed to a sound and whether

\footnotetext{
7 "[...] auditory hallucinations are by far the most common and characteristic of Schizophrenia." [1] pp. 275

8 These are arguable translations from French adjectives: familier, rassurant, plaisant, bizarre, angoissant, envahissant
} 
there are similarities between brain processing of sounds and words. For that, a priming protocol was used with word/sound pairs and the degree of congruence between the prime and the target was manipulated. To design stimuli, seventy abstract sounds from the nine "balanced" (see section 2) categories of Schaeffer's typology table were evaluated in a pre-test to define the word/sound pairs. The sounds were presented successively to listeners who were asked to write the first words that came to their mind after listening. A large variety of words were given by listeners. One of the sounds obtained for instance the following responses: "dry, wildness, peak, winter, icy, polar, cold". Nevertheless, for most sounds, it was possible to find a common word that was accepted as coherent by more than $50 \%$ of the listeners. By associating these common words with the abstract sounds, we designed forty-five related word/sound pairs. The non-related pairs were constructed by recombining words and sounds randomly. This step allowed us to validate the abstract sounds since no label referring to the actual source was given. Indeed when listeners are asked to explicitly label abstract sounds, different labels that were more related to the sound quality were collected. In a first experiment a written word (prime) was visually presented before a sound (target) and subjects had to decide whether or not the sound and the word fit together. In a second experiment, presentation order was reversed (i.e. sound presented before word). Results showed that participants were able to evaluate the semiotic relation between the prime and the target in both sound-word and word-sound presentations with relatively low inter-subject variability and good consistency (see [32] for details on experimental data and related analysis). This result indicated that abstract sounds are suitable for studying conceptual processing. Moreover, their contextualization by the presentation of a word reduced the variability of interpretations and led to a consensus between listeners. The study also revealed similarities in the electrophysiological patterns (Event Related Potentials) between abstract sounds and word targets, supporting the assumption that similar processing is involved for linguistic and non-linguistic sounds.

\subsection{Sound synthesis}

Intuitive control of synthesizers through high-level parameters is still an open problem in virtual reality and sound design. Both in industrial and musical contexts, the challenge consists of creating sounds from a semantic description of their perceptual correlates. Indeed, as discussed formerly, abstract sounds can be rich from an acoustic point of view and enable testing of different spectrotemporal characteristics at the same time. Thus they might be useful to identify general signal properties characteristic of different sound categories. In addition, they are particularly designed for restitution through speakers (as this is the case for synthesizers). For this purpose, we proposed a general methodology based on evaluation and analysis of abstract sounds aiming at identifying perceptually relevant signal characteristics and propose an intuitive synthesis control. Given a set of desired control parameters and a set of sounds, the proposed method 
consists of asking listeners to evaluate the sounds on scales defined by the control parameters. Sounds with same/different values on a scale are then analyzed in order to identify signal correlates. Finally, using feature based synthesis [20], signal transformations are defined to propose an intuitive control strategy.

In [23], we addressed the control of perceived movement evoked by monophonic sounds. We first conducted a free categorization task asking subjects to group sounds that evoke a similar movement and to label each category. The aim of this method was to identify sound categories to further identify perceptually relevant sound parameters specific to each category. Sixty-two abstract sounds were considered for this purpose. Based on subjects' responses, we identified six main categories of perceived movements: "rotate", "fall down", "approach", "pass by", "go away" and "go up" and identified a set of sounds representative of each category. Note that like in the previous studies, the labels given by the subjects did not refer to the sound source but rather to an evocation. Based on this first study, we aimed at refining the perceptual characterization of movements and identify relevant control parameters. For that, we selected 40 sounds among the initial corpus of 200 sounds. Note that in the case of movement, we are aware that the recognition of the physical sound source can introduce a bias in the evaluation. If the source can be easily identified, the corresponding movement is more likely to be linked to the source: a car sound only evokes horizontal movement and cannot fall or go up. Thus, we asked 29 listeners to evaluate the 40 sounds through a questionnaire including the two following questions rated on a linear scale:

- "Is the sound source recognizable?" (rated on a non graduated scale from "not recognizable" to "easily recognizable")

- "Is the sound natural?" (rated from "natural" to "synthetic")

When the sources were judged "recognizable", listeners were asked to write a few words to describe the source.

We found a correspondence between responses of the two questions: the source is perceived natural as long as it is easily recognized $(\mathrm{R}=.89)$. Note that abstract sounds were judged as "synthesized" sounds even if they actually were recordings from vibrating bodies. Finally we asked listeners to characterize the movements evoked by sounds with a drawing interface that allowed representing combination of the elementary movements previously found (sounds can rotate and go-up at the same time) and where drawing parameters correspond to potential control parameters of the synthesizer. Results showed that it was possible to determine the relevant perceptual features and to propose an intuitive control strategy for a synthesizer dedicated to movements evoked by sounds.

\section{Conclusion}

In this paper, we presented the advantages of using abstract sounds in audio and perception research based on a review of studies in which we exploited their distinctive features. The richness of abstract sounds in terms of their acoustic 
characteristics and potential evocations open various perspectives. Indeed, they are generally perceived as "unrecognizable", "synthetic" and "bizarre" depending on context and task and these aspects can be relevant to help listeners to focus on the intrinsic properties of sounds, to orient the type of listening, to evoke specific emotions or to better investigate individual differences. Moreover, they constitute a good compromise between elementary and ecological stimuli.

We addressed the design of the sound corpus and of specific procedures for listening tests using abstract sounds. In auditory perception research, sound categories based on well identified sound sources are most often considered (ver$\mathrm{bal} /$ non verbal sounds, environmental sounds, music). The use of abstract sounds may allow defining more general sound categories based on other criteria such as listeners' evocations or intrinsic sound properties. Based on empirical researches from electroacoustic music trends, the sound typology proposed by P. Schaeffer should enable the definition of such new sound categories and may be relevant for future listening tests including any sound. Otherwise, since abstract sounds convey multiple information (attribution of several meanings), the procedure is of importance to orient type of listening towards the information that actually is of interest for the experiment.

Beyond these considerations, the resulting reflections may help us to address more general and fundamental questions related to the determination of invariant signal morphologies responsible for evocations and to which extent "universal" sound morphologies that do not depend on context and type of listening exist.

\section{References}

1. Association, A.P.: The Diagnostic and Statistical Manual of Mental Disorders, Fourth Edition (DSM-IV). American Psychiatric Association, http://www.psychiatryonline.com/DSMPDF/dsm-iv.pdf (1994) (Last viewed, February 2011)

2. Ballas, J.A.: Common factors in the identification of an assortment of brief everyday sounds. Journal of Experimental Psychology: Human Perception and Performance 19 (1993) $250-267$

3. Bentin, S., McCarthy, G., Wood, C.C.: Event-related potentials, lexical decision and semantic priming. Electroencephalogr Clin Neurophysiol 60 (1985) 343-355

4. Bergman, P., Skold, A., Vastfjall, D., Fransson, N.: Perceptual and emotional categorization of sound. The Journal of the Acoustical Society of America 126 (2009) 3156-3167

5. Bloit, J., Rasamimanana, N., Bevilacqua, F.: Towards morphological sound description using segmental models. In: DAFX, Milan, Italie (2009)

6. Bonebright, T.L., Miner, N.E., Goldsmith, T.E., Caudell, T.P.: Data collection and analysis techniques for evaluating the perceptual qualities of auditory stimuli. ACM Trans. Appl. Percept. 2 (2005) 505-516

7. Bonebright, T.L., Nees, M.A.: Most earcons do not interfere with spoken passage comprehension. Applied Cognitive Psychology 23 (2009) 431-445

8. Bregman, A.S.: Auditory Scene Analysis. The MIT Press (1990) 
9. Chion, M.: Audio-vision, Sound on Screen. Columbia University Press, New-York (1993)

10. Cross, L.: Electronic music, 1948-1953. Perspectives of New Music (1968)

11. Dack, J.: Abstract and concrete. Journal of Electroacoustic Music 14 (2002)

12. Dack, J., North, C.: Translating pierre schaeffer: Symbolism, literature and music. In: Proceedings of EMS06 Conference, Beijing. (2006)

13. Dessein, A., Lemaitre, G.: Free classification of vocal imitations of everyday sounds. In: Sound And Music Computing (SMC 09), Porto, Portugal (2009) 213-218

14. Dubois, D., Guastavino, C., Raimbault, M.: A cognitive approach to urban soundscapes: Using verbal data to access everyday life auditory categories. Acta Acustica united with Acustica 92 (2006) 865-874(10)

15. Eimert, H.: What is electronic music. Die Reihe 1 (1957)

16. Fastl, H.: Neutralizing the meaning of sound for sound quality evaluations. In: Proc. Int. Congress on Acoustics ICA 2001, Rome, Italy. Volume 4 CD-ROM. (2001)

17. Gaver, W.W.: How do we hear in the world ? explorations of ecological acoustics. Ecological Psychology 5 (1993) 285 - 313

18. Gaver, W.W.: What in the world do we hear ? an ecological approach to auditory source perception. Ecological Psychology 5 (1993) 1-29

19. Hermann, T.: Taxonomy and definitions for sonification and auditory display, Paris, France (2008) in proceedings.

20. Hoffman, M., Cook, P.R.: Feature-based synthesis: Mapping acoustic and perceptual features onto synthesis parameters. In: Proceedings of the 2006 International Computer Music Conference (ICMC), New Orleans. (2006)

21. Jekosch, U.: 8. In: Assigning Meaning to Sounds - Semiotics in the Context of Product-Sound Design. J. Blauert (2005) 193-221

22. McKay, C., McEnnis, D., Fujinaga, I.: A large publicly accessible prototype audio database for music research. (2006)

23. Merer, A., Ystad, S., Kronland-Martinet, R., Aramaki, M.: Semiotics of sounds evoking motions: Categorization and acoustic features. In Kronland-Martinet, R., Ystad, S., Jensen, K., eds.: CMMR 2007. Sense of Sounds. Springer, LNCS (2008) $139-158$

24. Micoulaud-Franchi, J.A., Cermolacce, M., Vion-Dury, J.: Bizzare and familiar recognition troubles of auditory perception in patient with schizophrenia. in preparation (2010)

25. Moore, B.C.J., Tan, C.T.: Perceived naturalness of spectrally distorted speech and music. The Journal of the Acoustical Society of America 114 (2003) 408-419

26. Peeters, G., Deruty, E.: Automatic morphological description of sounds. In: Acoustics 08, Paris, France (2008)

27. Ricard, J., Herrera, P.: Morphological sound description computational model and usability evaluation. In: AES 116th Convention. (2004)

28. Risset, J.C., Wessel, D.L.: Exploration of timbre by analysis and synthesis. In Deutsch, D., ed.: The psychology of music. Series in Cognition and Perception. Academic Press (1999) 113-169

29. Schaeffer, P.: Traité des objets musicaux. Editions du seuil (1966)

30. Schaeffer, P., Reibel, G.: Solfège de l'objet sonore. INA-GRM (1967)

31. Schlauch, R.S.: 12 - Loudness. In: Ecological Psychoacoustics. Elsevier Academic press (2004) 318-341

32. Schön, D., Ystad, S., Kronland-Martinet, R., Besson, M.: The evocative power of sounds: Conceptual priming between words and nonverbal sounds. Journal of Cognitive Neuroscience 22 (2010) 1026-1035 
33. Shafiro, V., Gygi, B.: How to select stimuli for environmental sound research and where to find them. Behavior Research Methods, Instruments, \& Computers 36 (2004) 590-598

34. Smalley, D.: Defining timbre - refining timbre. Contemporary Music Review 10 (1994) $35-48$

35. Smalley, D.: Space-form and the acousmatic image. Org. Sound 12 (2007) 35-58

36. Tanaka, K., Matsubara, K., Sato, T.: Study of onomatopoeia expressing strange sounds : Cases of impulse sounds and beat sounds. Transactions of the Japan Society of Mechanical Engineers. C 61 (1995) 4730-4735

37. Thoresen, L., Hedman, A.: Spectromorphological analysis of sound objects: an adaptation of pierre schaeffer's typomorphology. Organised Sound 12 (2007) 129141

38. Zeitler, A., Ellermeier, W., Fastl, H.: Significance of meaning in sound quality evaluation. Fortschritte der Akustik, CFA/DAGA 4 (2004) 781-782

39. Zeitler, A., Hellbrueck, J., Ellermeier, W., Fastl, H., Thoma, G., Zeller, P.: Methodological approaches to investigate the effects of meaning, expectations and context in listening experiments. In: INTER-NOISE 2006, Honolulu, Hawaii. (2006) 